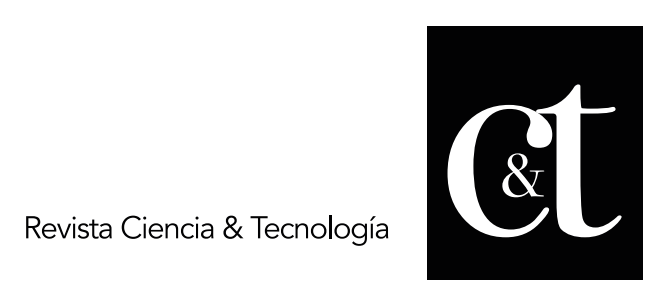

\title{
Probabilidad de ser víctima de violencia doméstica para las mujeres que laboran en el Perú
}

\section{Likelihood of being a victim of domestic violence for women who work in Peru}

\author{
Claudia Lucía Fátima Andía Vera ${ }^{1}$ \\ claudiaandiav@gmail.com \\ https://orcid.org/0000-0002-2846-6326 \\ Ricardo N. Villamonte Blas ${ }^{2}$ \\ rvillamonteb@unmsm.edu.pe \\ https://orcid.org/0000-0002-0759-9074
}

Recibido: 28/6/2021, Aceptado: 28/9/2021

\begin{abstract}
RESUMEN
En el Perú el género femenino participa cada vez más en el ámbito laboral remunerado. Sin embargo la violencia domestica contra la mujer se mantiene en una sociedad poco inclusiva. Es lenta la aplicación de derechos para las mujeres y aun predomina las oportunidades para varones.

La investigación tiene como objetivo, medir la probabilidad de ocurrencia de que una mujer peruana entre 18 y 59 años sea víctima de algún tipo de violencia doméstica cuando está empleada y sea beneficiaria del programa Quali Warma, utilizando un modelo econométrico logit multinomial .Los resultados concluyen que para una mujer que labora entre 18 y 59 años existe los siguientes niveles de probabilidad : $32.21 \%$ que sea víctima de violencia domestica por parte de su pareja, del $10.05 \%$ que sea víctima de violencia patrimonial, del $9.25 \%$ que sea víctima de violencia psicológica, $6.29 \%$ de ser víctima de violencia física y finalmente $6.61 \%$ de ser víctima de violencia sexual. Finalmente en el caso que no laboren y sean beneficiarias del programa QaliWarma la probabilidad de ser víctima de violencia domestica es de $67.79 \%$.
\end{abstract}

Palabras clave: violencia doméstica, probabilidad, modelo logit

\section{ABSTRACT}

In Peru, the female gender participates more and more in the paid work environment. However, domestic violence against women remains in a not very inclusive society. The application of rights for women is slow and opportunities for men still predominate.

\footnotetext{
${ }^{1}$ Economista. Universidad de Lima, Perú.

${ }^{2}$ Doctor en Ciencias Económicas. Universidad Nacional Mayor de San Marcos, Perú
} 


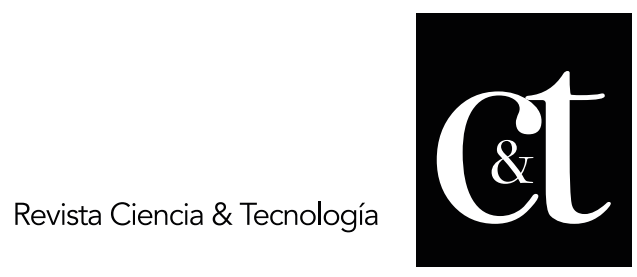

No. 32, 31 de octubre de 2021

ISSN impreso: 1390 - 6321

ISSN online: 2661 - 6734

The objective of the research is to measure the probability of a Peruvian woman between 18 and 59 years old being a victim of some type of domestic violence when she is employed and is a beneficiary of the Quali Warma program, using a multinomial logit econometric model. The results conclude that for a woman who works between 18 and 59 years there are the following probability levels: $32.21 \%$ that she is a victim of domestic violence by her partner, $10.05 \%$ that she is a victim of patrimonial violence, and $9.25 \%$ that she is a victim of violence psychological, $6.29 \%$ of being a victim of physical violence and finally $6.61 \%$ of being a victim of sexual violence. Finally, in the case that they do not work and are beneficiaries of the QaliWarma program, the probability of being a victim of domestic violence is $67.79 \%$.

Keywords: domestic violence, probability, logit model

\section{Introducción}

Según la Organización Mundial de la Salud (OMS)(2013), la violencia contra la mujer está considerada como un verdadero problema de salud pública, además de ser una violación flagrante de los derechos humanos de la mujer. El 35\% de las mujeres a nivel mundial han sido víctimas de violencia física y/o sexual por parte de su pareja o de otra persona, aunque las mujeres pueden estar expuestas a distintas formas de violencia. Concluye que ello configura un enorme problema no solo en la sociedad, sino que es un problema de salud pública mundial, en definitiva, es necesario una intervención a nivel mundial: Una vida sin violencia es un derecho humano fundamental, al que deben aspirar todos los hombres, mujeres y niños.

La Organización Mundial de la Salud (2017), a un nivel multipaís sobre salud de la mujer y violencia doméstica, ausculto 15 entornos en 10 países, siendo el Perú uno de ellos, se comprobó que entre el $10 \%$ y $52 \%$ de las mujeres han sufrido maltrato físico por parte de su pareja en algún momento de su vida, y un estimado entre el $10 \%$ y el 27 de violación sexual siendo niñas o adultas. Las conclusiones fueron vastas y extendidas, evidenciándose altos niveles de violencia sexual contra las mujeres y niñas, lo cual conllevo a que se presentaran 14 recomendaciones bien sustanciadas sobre cómo debe abordarse dicha problemática.

Según la Organización de las Naciones Unidas (como se citó en Andía, 2020) se define violencia como todo aquel acto de violento al sexo femenino que tenga o pueda tener como resultado un daño o sufrimiento físico, sexual o psicológico, así como las amenazas de tales actos, la coacción o la privación arbitraria de la libertad, tanto si se producen en la vida pública como en la vida privada ( p. 1 ).

En el Perú en materia de violencia doméstica contra la mujer se vienen incorporando en la normativa legal importantes cambios pero los resultados esperados aún son lentos. Según el Instituto Nacional de Estadística e Informática (INEI), en el 2019 en el Perú, el 57.7\% de mujeres entre 12 y 49 años han sido víctimas de algún tipo de violencia por lo menos una vez en su vida por parte de su pareja. 


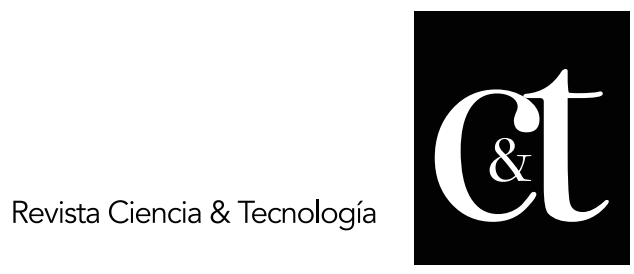

No. 32, 31 de octubre de 2021

ISSN impreso: 1390 - 6321

ISSN online: 2661 - 6734

Del total de mujeres víctimas de violencia el $58,2 \%$ no se encontraban laborando en el momento de la realización de la encuesta (INEI, 2017) y según el Ministerio de la Mujer y Poblaciones Vulnerables el $23 \%$ de mujeres trabajadoras remuneradas son víctima de violencia ejercida por su pareja en promedio 4 veces al año (2017). Por otro lado, en publicación realizada por Programa Nacional Aurora (2020), en sus informes estadísticos del Centro de Emergencia Mujer (CEM), al cierre del 2019, del total de casos registrados de violencia domestica para personas entre los 18 y 59 años, se registraron un total de 115,246 denuncias relacionadas a violencia doméstica contra la mujer en este rango de edad, un $37.5 \%$ más respecto a los registrados en el año 2018.

El presente estudio a partir de los enfoques teóricos de varios autores seleccionados así como de diferentes estudios aplicados, focaliza su interés en evaluar la probabilidad de varios tipos de violencia domestica de las mujeres beneficiarias del Programa Nacional de Alimentación Escolar Qali Warma, con el objetivo de lograr una evaluación para dicho programa nacional el cual es de especial interés en la lucha contra la exclusión social. El mencionado programa tiene como finalidad brindar un servicio de calidad cumpliendo los siguientes objetivos: garantizar el servicio alimentario durante todos los días del año escolar a los usuarios del programa de acuerdo a sus características y las zonas donde viven; contribuir a mejorar la atención de los usuarios del programa en clases, favoreciendo su asistencia y permanencia; promover mejores hábitos de alimentación en los usuarios del programa (MINDES , 2019)

\section{Revisión de la literatura}

Walker (1984) explica claramente que cuando la mujer ha experimentado la violencia, queda entonces incapacitada en el control de su voluntad con el paso del tiempo, lapso en el cual "se desarrolla la condición de impotencia aprendida, siendo ésta condición la responsable de la deficiencia cognoscitiva emocional y conductual que se puede observar en las mujeres maltratadas, ya están afectadas de forma negativa y se les retiene en esa relación abusiva" ( p. 161).

Bandura (1987), explica que el origen de la conducta violenta "está influida por la confluencia de factores biológicos, la experiencia directa y el aprendizaje observacional, siendo este último el más relevante, y plantea un determinismo reciproco entre esos tres factores". (p. 121). Explica que, en primer lugar, están presentes los factores genéticos y hormonales que influyen directamente en el desarrollo físico, el cual a su vez incide en la conducta del sujeto. En segundo lugar, la experiencia ayuda a facilitar las nuevas conductas, aunque en esencia lo que mueve la conducta humana proviene de la dotación natural y en tercer lugar, los procesos de aprendizaje vienen a constituir factores mediadores para adquirir el conocimiento social necesario en relación al manejo de la violencia, que tienen su inicio en la observación, donde se imitan los comportamientos e instrucciones que provienen directamente de los padres, lo cual determina el origen del esquema que copiaran los niños y permitirán incorporan en su modelo de comportamiento a futuro" ( p. 121). 


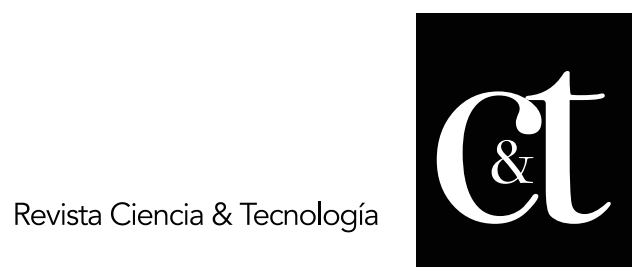

No. 32, 31 de octubre de 2021

ISSN impreso: 1390 - 6321

ISSN online: 2661 - 6734

Olaiz et al. (2006) explican que la identificación y la medición de la violencia constituyen un fenómeno complejo en consideración a los diversos tipos de violencia que existen y a la manera en que las mujeres mismas la perciben y la notifican. La violencia es un problema importante de salud, tanto por la alta prevalencia como por sus consecuencias inmediatas y acumulativas sobre la salud. Los resultados indican la necesidad urgente de prevenir y atender este problema.

VandeWeerd et al. (2011) comentan que la mayoría de las mujeres han sido víctimas de violencia ejercida por su pareja la menos una vez en su vida, ya sea psicológica o físicamente e identifica las siguientes variables que guardan relación e influyen en violencia y su nivel de incidencia demográficas, social y económica.

Karim y Razzaque (2007) en su estudio utilizando un modelo logístico encuentra que las mujeres mujer que participan en actividades que generen ingresos fuera del hogar tienen $29.8 \%$ más probabilidades de ser víctimas de violencia. Concluye que la educación y las condiciones socioeconómicas son cruciales para reducir el riesgo de violencia.

Olate et al., (2011), abordaron la relación que existe entre violencia en contra de las mujeres a nivel intrafamiliar y la incorporación de las mujeres al mercado laboral con el objetivo estimar el impacto de la tasa de participación laboral femenina en el número de denuncias de violencia intrafamiliar. Concluyen que no hay relación de causalidad directa entre la participación laboral femenina y el número de denuncias por violencia intrafamiliar, a pesar de los diferentes métodos de estimación empleados.

Dulcey, I. (2015), aplica un modelo probit para el caso colombiano, concluye que las mujeres con mayor propensión a ser víctima de algún tipo de violencia son las que no viven con su pareja, las que pertenecen a un nivel bajo de riqueza, las que no completaron secundaria, las que viven en zona urbana, las que trabajan, las que han vivido situaciones de violencia en su hogar de crianza.

Borrego y Carrasco (2017), utilizando un modelo probit concluyen, que el hecho de que la pareja masculina esté empleada desempeña un rol predominante en el riesgo de violencia física, mientras que el que una mujer esté empleada reducirá el riesgo de violencia solo si la pareja también está empleada. Hay menor riesgo a violencia domestica cuando ambos están empleados.

Eswaran y Malhotra (2011), investigaron de qué manera la violencia doméstica en los países en desarrollo impacta sobre la autonomía de la mujer para la toma de decisiones dentro del hogar, en el caso de la India ,utilizaron un modelo de probabilidad lineal .Los resultados hallados reflejan que de las mujeres que no trabajan para algún miembro de su familia, el $16 \%$ es víctima de violencia doméstica. De las mujeres que trabajan para algún miembro de su familia solo el $9 \%$ es víctima de violencia doméstica. 


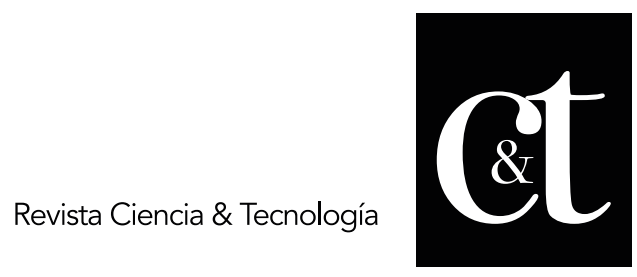

No. 32, 31 de octubre de 2021

ISSN impreso: 1390 - 6321

ISSN online: 2661 - 6734

Olivera (2017) mediante un modelo probit aprecia que la violencia infligida a las mujeres por parte de sus parejas, se convierte en un problema que, no solo las afecta a ellas como mujeres, sino que afecta a la dinámica familiar y por ende sus efectos se extienden a la comunidad y a la sociedad en general.

La probabilidad de que un niño trabaje aumenta si su madre es víctima de violencia en variadas ocasiones y también si es una violencia severa (p.35). En el estudio realizado por la Facultad de Derecho y Ciencia Política de la Universidad Ricardo Palma en el año 2017, se expone que la violencia contra la mujer es un problema de salud pública en el Perú. El estudio concluye que el $64 \%$ de las mujeres de Santiago de Surco encuestadas, indicaron haber sufrido de violencia emocional o psicológica, manifestándose ampliamente en situaciones de control, trato humillante y amenazas, lo cual vulnera el derecho a la libertad y autonomía de las mujeres. (URP, 2017)

Cataño, J. (2017), a través de su modelo probit concluye en su estudio para Colombia que,existe una relación positiva y estadísticamente significativa entre la pobreza multidimensional y la aceptación de la violencia domestica ejercida contra la mujer en Colombia(p.34).

El estudio realizado por Mendoza (2017) refleja que el grupo de mujeres que trabajan tienen de $14.2 \%$ a $38.4 \%$ más de probabilidades de ser víctimas de violencia domestica de tipo emocional en comparación a las mujeres que no trabajan (p. 51). El factor empleo en una mujer peruana no tiene el efecto de reducir la probabilidad de ser víctima de violencia doméstica, por el contrario, la vuelve más vulnerable frente a mujeres que no trabajan. Se revela que cuando una mujer es beneficiaria del programa Qali Warma tiene menores probabilidades de ser víctima de violencia doméstica en mujer que trabaja.

\section{Tipos de violencia contra la mujer}

Violencia económica patrimonial

Según la Procuraduría General de la Republica de México (2017) puede ser entendida como las acciones u omisiones que afectan la supervivencia de las víctimas; privándolas, ya sea de los recursos económicos necesarios para la manutención del hogar y la familia, o de bienes patrimoniales esenciales que satisfacen las necesidades básicas para vivir, como la alimentación, ropa, vivienda y el acceso a la salud (p.1).

Violencia emocional o psicológica

Según la Unidad de Igualdad de Genero (2017) Son actos que conllevan a la desvalorización y buscan disminuir o eliminar los recursos internos que la persona posee para hacer frente a las diferentes situaciones de su vida cotidiana (p.2 ).

La PCM (2015) la Ley N 30364 , en su artículo No 8, señala que es la acción o conducta que tiende a controlar o aislar a la persona contra su voluntad, así como de humillarla o avergonzarla, insultarla, estigmatizarla o estereotiparla, sin importar el tiempo que se requiera para su recuperación, ocasionando en 


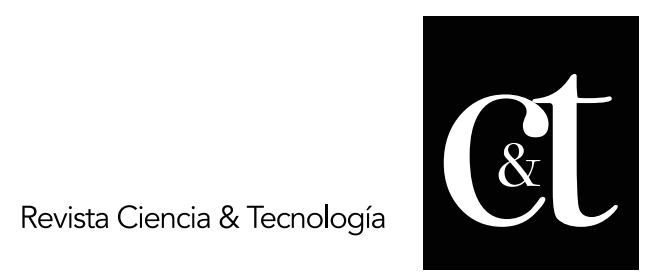

No. 32, 31 de octubre de 2021

ISSN impreso: 1390 - 6321

ISSN online: 2661 - 6734

consecuencia daños psíquicos, el cual afecta algunas funciones mentales o capacidades de la persona, como consecuencia de un conjunto de situaciones de violencia, causadas por agresiones temporales o permanentes, que podrían ser reversibles o irreversibles del funcionamiento integral de la víctima (p.5) .

Violencia física

Desde la perspectiva legal peruana, la violencia física es considerada como la acción o conducta que causa daños a la integridad corporal, así como también a la salud. Esta incluido el daño por negligencia, descuido o por privación de las necesidades básicas, que hayan ocasionado daño físico o que puedan llegar a ocasionarlo, sin tomar en cuenta el tiempo que se requiere para su rehabilitación (Ley $\mathrm{N}^{\circ} 30364$, art. $8^{\circ}$ ). Al respecto el reconocido catedrático español Ramón Agustina (2000), citado por (Castillo, 2018, p. 41), argumenta que la violencia física suele clasificarse según el tiempo que se requiere para su sanación, en estas categorías: levísimas, leves, moderada, grave y extrema (ocasiona la muerte).

Violencia sexual

La Ley $\mathrm{N}^{\circ} 30364$ en su artículo $8^{\circ}$, especifica que son acciones de naturaleza puramente sexual que son cometidas contra una persona sin su consentimiento o bajo coacción, están incluidos actos que no involucran penetración o contacto físico alguno.

\section{Métodos}

Se trabajo con una muestra de la data extraída del Programa AURORA, que emite los informes y estadísticas relacionadas a violencia doméstica, atendidos a través de los Centros de Emergencia Mujer (CEM) de mujeres entre los 18 y 59 años. La muestra se aplico a nivel descentralizado, y se distribuiyo tomando en cuenta los niveles de participación regional de casos de violencia doméstica según el reporte CEM 2019. La muestra fue de 1,067 personas.

La hipótesis general de trabajo considera que : existe probabilidad de que una mujer peruana entre los 18 y 59 años y beneficiaria del programa Qali Warma sea víctima de algún tipo de violencia domestica cuando esta se encuentra empleada. Las hipótesis especificas se refieren a que hay también probabilidad de violencia: económica patrimonial, psicológica, física, y sexual . Finalmente la probabilidad es menor cuando no trabaja y es beneficiaria del programa Qali Warma.

El modelo econométrico que a continuación se utilizo se fundamenta en los siguientes autores : Mendoza (2017), VandeWeerd et al., (2011), Olate et al, (2011), Eswaran y Malhotra (2011).

El modelo seleccionado es un logit multinomial pues se quiere medir la probabilidad de la variable " $Y$ " dada la variable " $X$ ", usando variables dummy . Se asume que $Y$ tiene una distribución multinomial. Permite medir la probabilidad de cambio en las variables cuando estas están expuestas a diferentes condiciones. En este caso, frente a la condición laboral de la mujer se mide la probabilidad de ser víctima de algún tipo de violencia domestica definidos dentro del modelo. 
La teoría del modelo considera los siguientes elementos: Dentro del modelo estimado existen $m$ categorías, donde $\mathrm{p}_{j}(j=1,2, \ldots m$ probabilidades $)$, la idea del modelo logit multinomial de una forma binaria, brinda la probabilidad de escoger p Donde:

$$
\begin{aligned}
\frac{p_{1}}{p_{1}+p_{m}} & =F\left(\beta_{1} X\right) \\
\frac{p_{2}}{p_{2}+p_{m}} & =F\left(\beta_{2} X\right) \\
\frac{p_{m}}{p_{m-1}+p_{m}} & =F\left(\beta_{m-1} X\right)
\end{aligned}
$$

Por tanto, esto conlleva a que:

$$
\begin{gathered}
\frac{p_{j}}{p_{m}}=\frac{F\left(\beta_{j} X\right)}{1-F\left(\beta_{j} X\right)}=G\left(\beta_{j} X\right) ; \\
\text { donde } j=1,2, \ldots m-1
\end{gathered}
$$

Tenemos (1):

$$
\left[1+\sum_{j=1}^{m-1} \frac{p_{j}}{p_{m}}\right]^{-1}
$$

Entonces (2):

$$
p_{j}=\frac{G\left(\beta_{j} X\right)}{1+\sum_{j=1}^{m-1} \frac{p_{j}}{p_{m}}}
$$

Se considera que se obtienen observaciones de distribución multinomial según las probabilidades en (1) y (2). Desde un punto de vista operacional la distribución logística para el error hace que $G\left(\beta_{j} X\right)=\exp G\left(\beta_{j} X\right)$, por tanto:

$$
p_{j}=\frac{e^{\beta_{j} X} G}{D}
$$

Donde $j=1,2, \ldots \mathrm{m}-1$ 


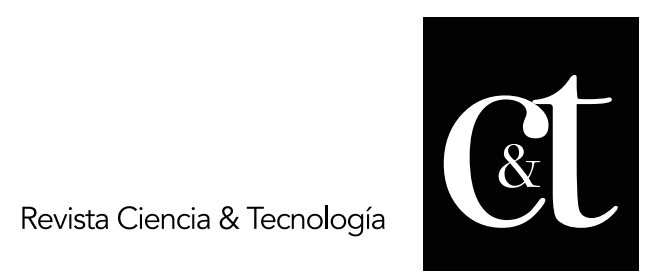

No. 32, 31 de octubre de 2021

ISSN impreso: 1390 - 6321

ISSN online: 2661 - 6734

Con

$$
p_{m}=1+\sum_{j=1}^{m-1} \frac{p_{j}}{p_{m}} e^{\beta_{j} X}
$$

De esta forma se define el modelo logit multinomial que se representa de la siguiente forma:

$$
\begin{gathered}
P(Y=j \mid X)=\frac{e\left(X \beta_{j}\right)}{1+\sum_{h=1}^{j} e(X \beta h)}=\operatorname{Pj}(x, \beta) \\
\text { para } j=1,2, \ldots j \\
\operatorname{Pr}_{i j}=\operatorname{Pr}\left(y_{i}=j\right)=\frac{\exp \left(x_{j} \beta_{j}\right)}{\sum_{h=1}^{j} \exp \left(x_{j} \beta_{k}\right)}
\end{gathered}
$$

Donde:

e = número de Euler, a la potencia

$(i)=$ muestra aleatoria

$\mathrm{Y}=$ variable dependiente

La variable dependiente $\left(Y_{i}\right)$ toma valores entre 1 y 4 dependiendo del tipo de violencia incurrida, la elección del agente estará en función a las variables explicativas $(X)$, las cuales representan características personales, como edad, sexo, situación laboral, y si es o no beneficiario de QaliWarma. Si $\left(Y_{i j}\right)$ es el tipo de violencia que representa para el sujeto, el supuesto del modelo se expresará como:

$$
w_{n} X_{i j}^{\prime}=\beta+\varepsilon_{i j} \text {, donde } j=1, \ldots, 4
$$

Si se asume la opción $j$, se asume que $U_{i j}$ es el conjunto de los tipos de violencia posibles, por tanto, es necesario calcular cual es la probabilidad de que $j$ sea seleccionada, es decir

$$
\text { Prob }\left(U_{i j}>U_{i k}\right) \text { para todo } k \text {, distinto de } j
$$


Este cálculo de probabilidad depende de la distribución del término estocástico que representa $\varepsilon_{i j}$. se debe suponer que es una distribución logística. Si se dispone de información para cada individuo, se da la probabilidad:

$$
\begin{gathered}
\operatorname{Prob}\left(u_{i j}>U_{i k}=\operatorname{Prob}\left(y_{i}=j\right)=\exp \left(\beta_{j} X_{i}\right) / n_{k-1}^{4} \exp \left(\beta_{j} X_{i}\right)\right. \\
j=1, \ldots, 4
\end{gathered}
$$

$Y_{i}$ indica el tipo de violencia que ha sufrido la victima $(i), \beta$ representa el coeficiente a estimar y $X_{i}$ son las características de $i$.

Entonces la expresión general del modelo es:

$$
\begin{gathered}
\operatorname{Prob}\left(Y_{i}=0\right)=\frac{1}{1+\sum_{j=1}^{j-1} e^{\beta_{k j} X_{k j}}}, \text { para } j=0 \\
\operatorname{Prob}\left(Y_{i}=j\right)=\frac{e^{\beta_{k j} x_{k j}}}{1+\sum_{j=1}^{j-1} e^{\beta_{k j} X_{k j}}}, \text { para } j=1,2, \ldots(J-1)
\end{gathered}
$$

Las pruebas para el evaluar el adecuado tratamiento de la data son : ajuste del modelo -logaritmo de la verosimilitud y Chi Cuadrado, prueba de bondad de ajuste, prueba de contraste de verosimilitud.

Operacionalización del modelo: para la presente investigación se empleo un modelo basado en el modelo propuesto por (Mendoza A. , 2017), el cual tiene como variable dependiente yi la cual toma 4 posibles resultados entre 0 y 1 e indica si la mujer fue víctima de violencia algún tipo de violencia definida o no. Los parámetros beta tendrán tres valores para las betas (constante y para cada variable explicativa). En donde:

- Xi1 : Mujer trabaja o no

- Xi2: Hogar de la mujer es beneficiado por Qaliwarma o no

- $\beta 11=\beta 21=0$ : Parámetros de la primera alternativa $(j=1)$, permite solucionar el problema de identificación.

- $\quad \beta 12$ - $\beta 22$ : Parámetros de la segunda alternativa $(j=2)$

- $\quad \beta 13$ - $\beta 23$ : Parámetros de la tercera alternativa $(j=3)$

- $\quad \beta 14$ - $\beta 24$ : Parámetros de la cuarta alternativa $(j=4)$

Entonces la ecuación propuesta adquiere los siguientes elementos:

$$
\frac{1}{1+e^{\beta_{12}+\beta_{22} X_{i 1}+\beta_{32} X_{i 2}}+e^{\beta_{13}+\beta_{23} X_{i 1}+\beta_{33} X_{i 2}}+e^{\beta_{14}+\beta_{24} X_{i 1}+\beta_{34} X_{i 2}}}, j=1
$$




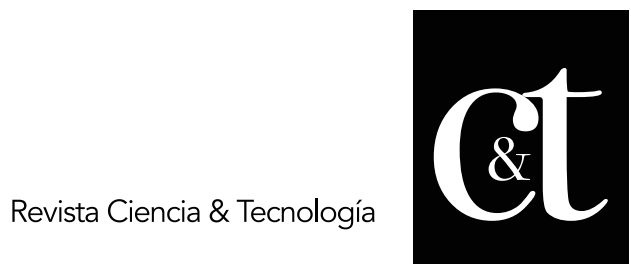

No. 32, 31 de octubre de 2021

ISSN impreso: 1390 - 6321

ISSN online: 2661 - 6734

$$
\begin{aligned}
& p_{i 2}=\frac{e^{\beta_{12}+\beta_{22} X_{i 1}+\beta_{32} X_{i 2}}}{1+e^{\beta_{12}+\beta_{22} X_{i 1}+\beta_{32} X_{i 2}}+e^{\beta_{13}+\beta_{23} X_{i 1}+\beta_{33} X_{i 2}}+e^{\beta_{14}+\beta_{24} X_{i 1}+\beta_{34} X_{i 2}}}, j=2 \\
& p_{i 1}=\frac{e^{\beta_{13}+\beta_{23} X_{i 1}+\beta_{33} X_{i 2}}}{1+e^{\beta_{12}+\beta_{22} X_{i 1}+\beta_{32} X_{i 2}}+e^{\beta_{13}+\beta_{23} X_{i 1}+\beta_{33} X_{i 2}}+e^{\beta_{14}+\beta_{24} X_{i 1}+\beta_{34} X_{i 2}}}, j=3 \\
& p_{i 1}=\frac{e^{\beta_{14}+\beta_{24} X_{i 1}+\beta_{34} X_{i 2}}}{1+e^{\beta_{12}+\beta_{22} X_{i 1}+\beta_{32} X_{i 2}}+e^{\beta_{13}+\beta_{23} X_{i 1}+\beta_{33} X_{i 2}}+e^{\beta_{14}+\beta_{24} X_{i 1}+\beta_{34} X_{i 2}}}, j=4
\end{aligned}
$$

La lógica del modelo considera que : existe relación entre la variable de nominal (xi): mujer que trabaja o no y la variable dependiente (yi): mujer es víctima de cualquiera los tipos de violencia definidos o no bajo condiciones individuales, sociales, económicas. se considera que tienen una distribución independiente. En este caso, frente a la condición laboral de la mujer se mide la probabilidad de ser víctima de algún tipo de violencia domestica definidos dentro del modelo (véase

\begin{tabular}{|c|c|c|}
\hline Variable & Definición & Indicador \\
\hline$Y_{i} 1$ & $\begin{array}{l}\text { Violencia Económica } \\
\text { Patrimonial }\end{array}$ & $\begin{array}{l}\text { Yi1 }=1 \text { Sí existe Violencia } \\
\text { Yi1 }=0 \text { No Existe }\end{array}$ \\
\hline$Y_{i} 2$ & Violencia Psicológica & $\begin{array}{l}\text { Yi2 }=1 \text { Sí existe Violencia } \\
\text { Yi2 }=0 \text { No Existe }\end{array}$ \\
\hline$Y_{i} 3$ & Violencia Física & $\begin{array}{l}\text { Yi3 }=1 \text { Sí existe Violencia } \\
\text { Yi3 }=0 \text { No Existe }\end{array}$ \\
\hline$Y_{i}$ i4 & Violencia Sexual & $\begin{array}{l}\text { Yi4=1 Sí existe Violencia } \\
\text { Yi4=0 No Existe }\end{array}$ \\
\hline$X_{i} 1$ & Mujer Trabaja o no & $\begin{array}{l}\text { Xi1=1 Sí trabaja } \\
\text { Xi1=0 No Trabaja }\end{array}$ \\
\hline$X_{i} 2$ & $\begin{array}{l}\text { Hogar Beneficiario del } \\
\text { Programa Social Qali } \\
\text { Warma }\end{array}$ & $\begin{array}{l}X i 2=1 \text { Sí es beneficiario } \\
\mathrm{Xi2}=0 \text { No es beneficiario }\end{array}$ \\
\hline
\end{tabular}
tabla No 1).

Tabla No 1 Variables e indicadores

Fuente: Elaboración propia

\section{Resultados y Discusión}

La significancia del modelo se evalúo mediante la prueba de significancia Chi cuadrado con un $95 \%$ de confiabilidad, arrojó una relación positiva continua entre las variables propuestas, el valor obtenido es 0.002 ( Véase Tabla 2), por tanto, el modelo de regresión logística multinomial se ajusta al conjunto de datos, como se muestra en la tabla a continuación. 
Tabla No 2 Ajuste del modelo - Logaritmo de la verosimilitud y Chi Cuadrado

\begin{tabular}{lcccc}
\hline & $\begin{array}{c}\text { Criterios } \\
\text { de ajuste de } \\
\text { modelo } \\
\text { Logaritmo } \\
\text { de la } \\
\text { verosimilitud } \\
-2\end{array}$ & $\begin{array}{c}\text { Chi- } \\
\text { cuadrado }\end{array}$ & gl & Sig. \\
& 108.866 & & & \\
vodelo & 84.604 & 24.262 & 8 & 0.002 \\
\hline $\begin{array}{l}\text { Sólo } \\
\text { intersección }\end{array}$ & Final & & & \\
\hline
\end{tabular}

Asimismo la bondad de ajuste tiene una significancia apropiada de 0.006 ( Véase Tabla $\mathrm{N}^{\circ} 3$ ) lo cual permite determinar que el modelo se ajusta de forma correcta a los datos ya que, las diferencias de los valores observados y predictivos del modelo son menores y no presentan sesgos.

Tabla No 3 Prueba de bondad de ajuste

\begin{tabular}{llll}
\hline & $\begin{array}{l}\text { Chi- } \\
\text { cuadrado }\end{array}$ & Gl & Sig. \\
\hline Pearson & 14.637 & 4 & 0.006 \\
Desvianza & 14.938 & 4 & 0.005 \\
\hline
\end{tabular}

Fuente: Encuesta de elaboración Propia

Respecto a las variables intervinientes para la prueba de verosimilitud, los resultados arrojaron que existe evidencia estadística para afirmar que la condición laboral de una mujer entre los 18 y 59 años si influye en el nivel de violencia doméstica. 
Tabla No 4 Pruebas de contraste de verosimilitud

\begin{tabular}{|c|c|c|c|c|}
\hline \multirow[b]{2}{*}{ Efecto } & $\begin{array}{ll}\text { Criterios } & \text { de } \\
\text { ajuste } & \text { de }\end{array}$ & \multicolumn{3}{|c|}{ Pruebas de la razón de verosimilitud } \\
\hline & $\begin{array}{ll}\text { de la } & \text { verosimilitud } \\
-2 & \text { de } \\
\text { modelo } & \\
\text { reducido } & \end{array}$ & $\begin{array}{l}\text { Chi- } \\
\text { cuadrado }\end{array}$ & gl & Sig. \\
\hline $\begin{array}{l}\text { Intersección } \\
\text { Programa }\end{array}$ & 169.607 & 85.002 & 4 & 0.000 \\
\hline $\begin{array}{l}\text { Juntos-Qali } \\
\text { Warma }\end{array}$ & 89.382 & 4.778 & 4 & 0.311 \\
\hline TRABAJA & 103.784 & 19.180 & 4 & 0.001 \\
\hline
\end{tabular}

Fuente: Elaboración Propia

Según su clasificación, se pudo comprobar que el modelo propuesto clasifica de forma correcta el $71.2 \%$ la información obtenida, lo cual se considera como un valor bueno de explicación.

Los resultados exponenciales se obtuvieron a través de la siguiente formula

Donde

$$
y_{v x}=\beta+\beta_{t}+\beta_{j}
$$

$y_{v x}=$ Tipo de violencia

$\beta=$ Beta del intercepto

$\beta_{t}=$ Beta de la variable trabajo

$\beta_{j}=$ Beta de la Variable Qali Warma

y fueron los siguientes:

- Violencia Económico Patrimonial:

$y_{v e p}=-2.240+0.053+0.278=0.1482521$

- Violencia Psicológica

- Violencia Física

$$
y_{v p s i}=-3.145+0.462+0.693=0.13654
$$

- Violencia Sexual

$$
y_{v f i s}=-2.289 \pm 0.332+0.243=0.0927741
$$

- Violencia Sexual

$$
y_{v s e x}=-3.097 \pm 0.214+0.983=0.0975408
$$

$\operatorname{Prob}\left(Y_{i}=0\right)=\frac{1}{1+0.1282521+0.13654+0.0927741+0.0975408}=0.677917$

Por lo tanto en la tabla N05 se puede apreciar que una mujer entre los 18 y 59 años que labora y es beneficiaria del programa Qali Warma tiene una 109 


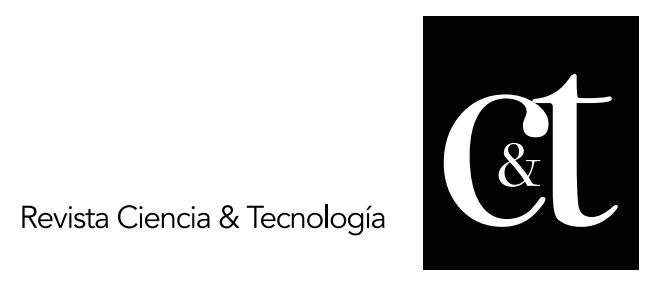

No. 32, 31 de octubre de 2021

ISSN impreso: 1390 - 6321

ISSN online: 2661 - 6734

probabilidad de $32.21 \%$ de que sea víctima de violencia doméstica, de $10.05 \%$ de que sea víctima de violencia domestica de tipo económico patrimonial, de $9.26 \%$ de que sea víctima de violencia doméstica de tipo psicológica, de $6.29 \%$ de que sea víctima de violencia domestica de tipo física, de $6.61 \%$ de que sea víctima de violencia domestica de tipo sexual, finalmente existe una probabilidad de que una mujer entre los 18 y 59 años que no labora y es beneficiaria del programa QaliWarma sea víctima de violencia doméstica en un $67.79 \%$ de probabilidad.

Tabla No 5 Tabla de Probabilidad

\begin{tabular}{|c|c|c|c|}
\hline violencia & e & probabilidad & $\begin{array}{c}\text { Tipo de } \\
\text { Probabilidad }\end{array}$ \\
\hline VIOLENCIA DOMESTICA & 0.677916907 & 0.32208309 & Media \\
\hline $\begin{array}{l}\text { VIOLENCIA } \\
\text { PATRIMONIAL }\end{array}$ & 0.148252097 & 0.1005026 & Baja \\
\hline VIOLENCIA PSICOLÓGICA & 0.13653999 & 0.09256277 & Baja \\
\hline VIOLENCIA FÍSICA & 0.092774107 & 0.06289314 & Baja \\
\hline VIOLENCIA SEXUAL & 0.097540843 & 0.06612459 & Baja \\
\hline NO TRABAJA & & 0.677916907 & Alta \\
\hline
\end{tabular}

Fuente: Elaboración Propia

De acuerdo a los resultados obtenidos en el Perú una mujer entre 18 y 59 años de edad y que esta en el programa Qali Warma tiene mayor probabilidad a ser víctima de violencia domestica sea esta de tipo económico patrimonial, psicológica, física o sexual cuando esta se encuentra trabajando. Esto va de acorde con las propuestas e investigaciones actuales que plantean que una mujer en situación laboral activa es más propensa a ser víctima de violencia doméstica de parte de su pareja(Mendoza, 2017). Los resultados de la presente investigación se relacionan con los de Mendoza (2017) quien concluye que existe una relación positiva entre los indicadores laborales y la violencia domestica femenina, y estos fluctúan entre el $14 \%$ y el $38 \%$ de probabilidades, dependiendo del tipo de violencia al que la víctima ha sido sometida. Estos resultados también se relacionan a los obtenidos por Olate et al., (2011), quien concluyó que las probabilidades de violencia femenina en los que puede incurrir una mujer cuando labora solo disminuyen en porcentajes mínimos las posibilidades de ocurrencia. En lo relacionado a la violencia física los resultados obtenidos se relacionan con los obtenidos por Dulcey, I. (2015) quien concluye que la probabilidad es de $8.4 \%$, Finalmente no se cumple la hipótesis de que cuando la mujer no trabaja y el hogar es beneficiado por el programa social, la probabilidad de ser víctima de violencia disminuye, caso contrario, el nivel de probabilidad es alto $68 \%$. 


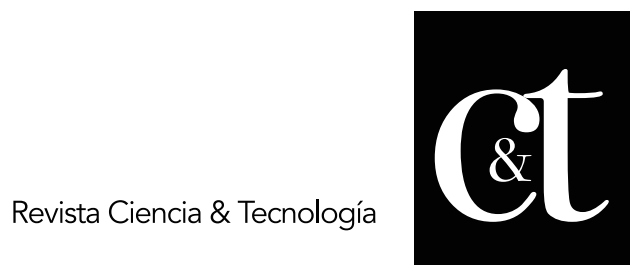

No. 32, 31 de octubre de 2021

ISSN impreso: 1390 - 6321

ISSN online: 2661 - 6734

El comportamiento desagregado por tipo de violencia dentro del periodo de análisis evidencia que no estamos por un buen camino a combatir este fenómeno, a pesar de los esfuerzos dentro del marco legal que se vienen realizando, como lo es la Ley $\mathrm{N}^{\circ} 30364$ y todas sus modificaciones las cuales tiene como objetivo principal la prevención, erradicación y sanciona miento a todo tipo de actos violentos en contra de la mujer (Poder Ejecutivo, 2015) la tendencia a ser víctima de alguno de los tipos de violencia no es negativa.

\section{Conclusiones}

La presente investigación ofrece una visión reveladora acerca de la relación causal y además del impacto probabilístico que existe entre la variable violencia domestica contra la mujer y la variable empleo a nivel nacional para todas las mujeres entre 15 y 49 años que participan en el programa Qali Warma. El análisis probabilístico ha permitido determinar en cuánto se incrementa la probabilidad de que una mujer sea víctima de violencia domestica bajo condiciones de estar trabajando o no. Constituye un aporte importante, en el contexto de que se pretende ser un país que alcance un desarrollo sostenible e igualitario en todos los sectores y niveles y a la vez sirve como crítica a la permanencia de ideas patriarcalistas, machistas, de ausencia de enfoque de género y falta de educación. La literatura revisada han sido de mucha utilidad para contextualizar el tema, reconocer factores, evaluar diferentes relaciones de probabilidades.

Nota. Los autores declaran que no existen conflictos de intereses, que no existe vínculo comercial, financiero o personal que pueda afectar el artículo o la institución editora-

\section{Referencias bibliográficas}

Andía C. (2021), Estudio sobre la relación entre participación laboral femenina y la probabilidad de ser víctima de violencia doméstica en el Perú 2009-2019, tesis para optar el título profesional de economista, Universidad de Lima. Perú.

Bandura, A. (1987). Teoría del aprendizaje social. Calpe. https://books.google.com.mx/books/about/Teor\%C3\%ADa_del_aprendizaje_s ocial.html?hl=es\&id=sJ-sQwAACAAJ

Cataño, J. (2017), Aceptación de la violencia domestica, pobreza y desigualdad: evidencia para Colombia. (Tesis de Maestría). Departamento de Economia, Universidad EAFIT. Medellin, Colombia. Disponible en: https://repository.eafit.edu.co/bitstream/handle/10784/13354/JoseLeonardo CatanoSanchez 2018. pdf? sequence $=$ 2\&isAllowed $=y$

Dulcey, I. (2015). Determinantes socioeconomicos de la violencia contra la mujer dentro de la pareja. un análisis del caso colombiano. Universidad Industrial de Santander. Facultad de Economia y Administración, Colombia. Obtenido de http://www.cedlas.econo.unlp.edu.ar/wp/wp-content/uploads/dulcey.pdf

Eswaran M, Malhotra N. (2011). Violencia doméstica y autonomía de la mujer en los países en desarrollo: Teoría y evidencia. Canadian Journal of Economics, 44, 1222-1263.

Obtenido

de https://onlinelibrary.wiley.com/doi/abs/10.1111/j.1540-5982.2011.01673.x 


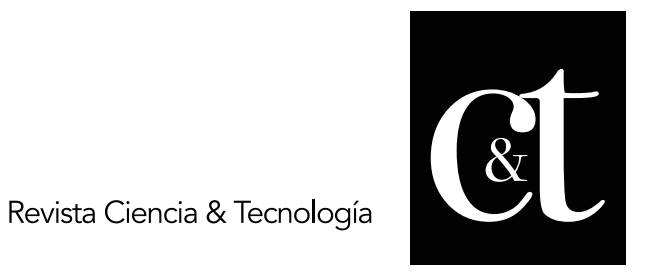

No. 32,31 de octubre de 2021

ISSN impreso: 1390 - 6321

ISSN online: 2661 - 6734

INEI. (2017). Encuesta Nacional sobre Relaciones Sociales 2017 https://www.inei.gob.pe/media/MenuRecursivo/noticias/nota-de-prensa-n21.

INEI. (2019). Encuesta Nacional sobre Relaciones Sociales 2019https://www1.inei.gob.pe/prensa/noticias/inei-presento-resultados-dela-encuesta-nacional-sobre-relaciones-sociales-2019-12304/

Karim M, Razzaque M. (2007). Violencia doméstica contra la mujer. Sus determinantes e implicaciones para la asignación de recursos de género. Obtenido de https://www.researchgate.net/publication/237581139_Domestic_Violence_ag ainst_Women_Its_Determinants_and_Implications_for_Gender_Resource_Allo cation

PCM (2015) Ley $N^{\circ}$ 30364, Ley para Prevenir, sancionar y erradicar la violencia contra las mujeres y los integrantes del grupo familiar. https://www.defensoria.gob.pe/deunavezportodas/wpcontent/uploads/2019/02/Ley3036 erradicarviolencia.pdf,

Mendoza, A. (2017). El efecto del empleo sobre la violencia doméstica: evidencia para las mujeres peruanas. Universidad de Piura. Obtenido de https://pirhua.udep.edu.pe/bitstream/handle/11042/3222/ECO-

L_005.pdf?sequence $=1$ \&isAllowed $=y$

Ministerio de Desarrollo e Inclusión Social (2019), Quiénes somos, Obtenido de : qaliwarma.gob.pe/quienes-somos

Olate C, Maffei T, Hernando A. (2011). Relacion entre empleo y violencia intrafamiliar. Ministerio de Planificacion MIDEPLAN. Gobierno de Chile. Obtenido de http://www.ministeriodesarrollosocial.gob.cl/btca/txtcompleto/mideplan/rela c.empleoviolenc.genero.pdf

Olaiz G, Rojas R, Valdez R, Franco A , Palma O.(2006). Prevalencia de diferentes tipos de violencia en usuarias del sector salud en México. http://www.scielo.org.mx/scielo.php?script=sci_arttext\&pid=S003636342006000800003

Olivera, G. (2017). Ensayo sobre violencia y capital humano: analisis con enfasis en economia de la familia. (Tesis Doctoral). Universidad Autonoma de Nuevo León. Facultad de Economía. Disponible en: http://eprints.uanl.mx/13945/1/1080216685.pdf

Organización Mundial de la Salud (2013). Estimaciones Mundiales y regionales de la violencia contra la mujer: prevalencia y efectos de la violencia conyugal y de la violencia sexual no conyugal en la salud. Disponible en: https://apps.who.int/iris/bitstream/handle/10665/85243/WHO_RHR_HRP_13 .06_spa.pdf?sequence $=1$ \&isAllowed $=y$

Organización Mundial de la Salud (2017). Estudio multipaís de la oms sobre salud de la mujer y violencia doméstica. Disponible en: https://apps.who.int/iris/bitstream/handle/10665/43390/924359351X_spa.p df?sequence $=1$ \&isAllowed $=y$

Procuraduría General de la Republica de Mexico (2017), Violencia patrimonial y económica contra lasmujeres en: https://www.gob.mx/cms/uploads/attachment/file/242427/6_Enterate_Viol encia_econo_mica_y_patrimonial_contra_las_mujeres_junio_170617.pdf

Programa Nacional Aurora,. (Junio de 2020). Fuente: https://portalestadistico.pe/boletines/. (C. d. (CEM), Ed.) Recuperado el 02 de Julio de 2020, de https://portalestadistico.pe/boletines/ 
UIG Procuraduria Genereal de la República PSICOLÓGICA CONTRA

de Mexico (2017) VIOLENCIA https://www.gob.mx/cms/uploads/attachment/file/253605/Violencia_psicol _gica_Mes_Agosto_2017_21-08-17.pdf

Universidad Ricardo Palma (2017). Violencia contra la mujer, en el distrito Santiago de Surco, Facultad de Derecho y Ciencia Política de la Universidad Ricardo Palma, 2017. Disponible http://repositorio.urp.edu.pe/bitstream/handle/urp/1080/Violencia\%20contr a\%20la\%20mujer\%20\%281\%29.pdf?sequence $=1$ \&isAllowed $=y$

VandeWeerd, C., Coulter, M., \& Mercado-Crespo, M. (2011). Las víctimas de violencia de pareja intima y la participación en la fuerza laboral. Obtenido de https://search.proquest.com/central/docview/881068354/fulltextPDF/32498 FF6EE5642D5PQ/1 ?accountid $=45277$

Walker Leonor, E. (1984). El síndrome de la mujer maltratada. Springer. https://latam.casadellibro.com/libro-el-sindrome-de-la-mujermaltratada/9788433026095/2062030 